\title{
Robust microbubble tracking for super resolution imaging in ultrasound
}

Hansen, Kristoffer B. ; Villagómez Hoyos, Carlos Armando; Brasen, Jens Christian; Diamantis, Konstantinos; Sboros, Vassilis; Sorensen, Charlotte Mehlin; Jensen, Jørgen Arendt

\section{Published in:}

Proceedings of 2016 IEEE International Ultrasonics Symposium

Link to article, DOI:

10.1109/ULTSYM.2016.7728793

Publication date:

2016

Document Version

Peer reviewed version

Link back to DTU Orbit

Citation $(A P A)$ :

Hansen, K. B., Villagómez Hoyos, C. A., Brasen, J. C., Diamantis, K., Sboros, V., Sorensen, C. M., \& Jensen, J. A. (2016). Robust microbubble tracking for super resolution imaging in ultrasound. In Proceedings of 2016 IEEE International Ultrasonics Symposium IEEE. https://doi.org/10.1109/ULTSYM.2016.7728793

\section{General rights}

Copyright and moral rights for the publications made accessible in the public portal are retained by the authors and/or other copyright owners and it is a condition of accessing publications that users recognise and abide by the legal requirements associated with these rights.

- Users may download and print one copy of any publication from the public portal for the purpose of private study or research.

- You may not further distribute the material or use it for any profit-making activity or commercial gain

- You may freely distribute the URL identifying the publication in the public portal 


\title{
Robust Microbubble Tracking for Super Resolution Imaging in Ultrasound
}

\author{
Kristoffer B. Hansen ${ }^{1}$, Carlos A. Villagómez-Hoyos ${ }^{1}$, Jens Christian Brasen ${ }^{1}$, \\ Konstantinos Diamantis ${ }^{2}$, Vassilis Sboros ${ }^{2}$, Charlotte Mehlin Sørensen ${ }^{3}$, and Jørgen Arendt Jensen ${ }^{1}$ \\ ${ }^{1}$ Department of Electrical Engineering, Technical University of Denmark, DK-2800 Lyngby, Denmark. \\ ${ }^{2}$ School of Engineering \& Physical Sciences, Heriot-Watt University, Edinburgh EH14 4AS, UK. \\ ${ }^{3}$ Section of Renal and Vascular Research, Department of Biomedical Sciences, \\ University of Copenhagen, DK-1165 København, Denmark.
}

\begin{abstract}
Currently ultrasound resolution is limited by diffraction to approximately half the wavelength of the sound wave employed. In recent years, super resolution imaging techniques have overcome the diffraction limit through the localization and tracking of a sparse set of microbubbles through the vasculature. However, this has only been performed on fixated tissue, limiting its clinical application. This paper proposes a technique for making super resolution images on non-fixated tissue by first compensating for tissue movement and then tracking the individual microbubbles. The experiment is performed on the kidney of a anesthetized Sprage-Dawley rat by infusing SonoVue at $0.1 \times$ original concentration. The algorithm demonstrated in vivo that the motion compensation was capable of removing the movement caused by the mechanical ventilator. The results shows that microbubbles were localized with a higher precision, reducing the standard deviation of the super localizations from $22 \mu \mathrm{m}$ to $8 \mu \mathrm{m}$. The paper proves that the restriction of completely fixated tissue can be eliminated, when making super resolution imaging with microbubbles.
\end{abstract}

\section{INTRODUCTION}

Super resolution algorithms using ultrasound are very appealing as they can overcome the diffraction limit, thus, providing enhanced visibility of vascular features. It can potentially make it possible to study the micro-vasculature and thereby directly the perfusion, of tissues and tumours.

The resolution of standard clinical ultrasound systems typically ranges between $50-500 \mu \mathrm{m}$ and are limited by its fundamental diffraction, which is determined by the operating frequency of 2-50 $\mathrm{MHz}$ for typical sonography. This range is able to visualize major organ structures and blood vessels, but fails at resolving micro-structures. Even high frequency ultrasound systems struggle to resolve micro-vessels with diameters around $100 \mu \mathrm{m}$ or less.

In the past years it has been proven possible to make super resolution images by the use of ultrasound and contrast agent, consisting of gas filled microbubbles (MBs) [1], [2]. MBs are enhanced in ultrasound images due to their non-linear properties and strong back-scattering ability. Individual bubbles can be tracked, if they are spatially isolated, and the exact position of each individual MB can be estimated by calculating the intensity weighted centroid of the Point Spread Function (PSF). Localization of thousands to millions of MBs makes it possible to achieve a resolution much smaller than the diffraction limit and introduces the opportunity to create super resolution images with current ultrasound systems.

In vivo experiments on a rat brain [1] and a fixated mouse ear [2] demonstrated that vessels with a diameter as narrow as $20 \mu \mathrm{m}$ could be detected, and two separate vessels only $20 \mu \mathrm{m}$ apart was distinguish inside the tissue $(>8 \mathrm{~mm})$. The results are great improvements when compared to the diffraction limit of $\sim 100 \mu \mathrm{m}$. However, these results were obtained by completely fixating the tissue, which limits the clinical application.

In a clinical setting, tissue moves during acquisition due to natural movement from respiration and pulse. Movement is a problem when tracking MBs through time, as the exact location of a vessel will be different in each frame. Motion compensation can be achieved by estimating the accumulated displacement and account for it during tracking.

In this paper, we propose a method for making super resolution images by combining motion compensation and $\mathrm{MB}$ tracking. The theory behind the method is described in Section II. An in vivo experiment has been performed on the kidney of a Sprage-Dawley rat. The experiment setup is described in Section III. Motion compensation was only performed in the axial direction due to the small lateral movement present in the experiment. The effect of motion compensation is shown by comparing tracking of MBs with and without compensation in Section IV. Lastly, two MB tracking images of the vasculature of the kidney is presented.

\section{THEORY}

\section{A. General Description}

The overall structure of the proposed method is illustrated in Fig. 1. The acquisition provides two set of data. Motion tracking is based on the B-mode image $\mathbf{I}_{t}(n, m, i)$, which is used to perform motion compensation on the contrast data. The contrast data $\mathbf{I}_{c}(o, p, i)$ contains only the response from the MBs. After compensation, noise reduction is performed to increase SNR from the bubbles. Finally, the bubble tracking is used to create a high resolution image. The data consist of images with $n, o$ rows in the axial direction, and $m, p$ columns 
in the lateral direction. The index $i$ refers to the frame number.

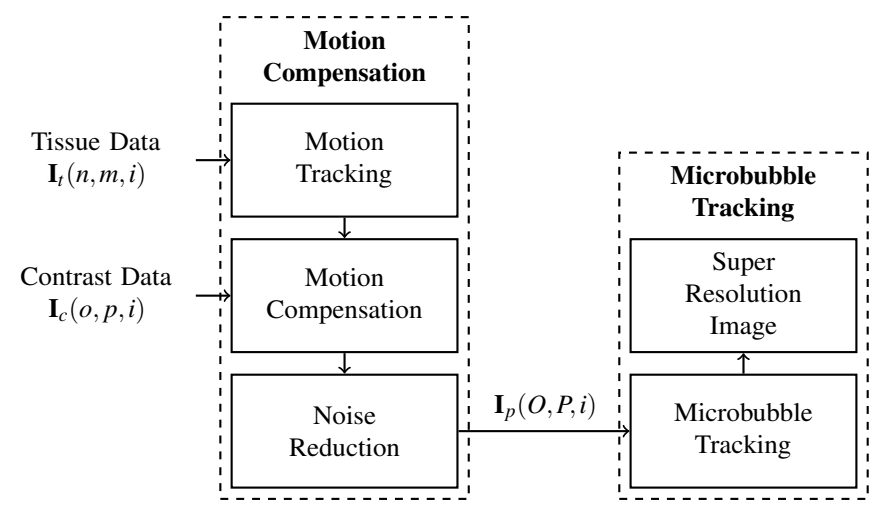

Fig. 1. Overall block diagram of the method. First, motion tracking, motion compensation and noise reduction are performed in "Motion Compensation" to prepare contrast images $\mathbf{I}_{c}(o, p, i)$ for MB tracking. The MB tracking algorithm is applied to the output $\mathbf{I}_{p}(O, P, i)$ and a super resolution image is created in "Microbubble Tracking".

\section{B. Motion Compensation}

Several different approaches to estimate movement and perform motion compensation have been investigated previously [3]-[7]. In the proposed method, RF cross-correlation is used to find the axial motion. Motion tracking is applied to beamformed and envelope detected tissue data from a B-mode sequence. The cross-correlation between a line segment in two following frames $i$ and $i+1$ at a location $(n, m)$ is given by:

$$
R(n, m, i, l)=\sum_{k_{n}=-K / 2}^{K / 2} I_{t}\left(n+k_{n}, m, i\right) \cdot I_{t}\left(n+k_{n}+l, m, i+1\right)
$$

where $K$ is the length of the line segment. The displacement is found at the lag $l$, which maximizes the correlation:

$$
d(n, m, i)=\arg \max _{l}\{R(n, m, i, l)\}
$$

Summing up the frame to frame movements $d(n, m, i)$ result in the absolute cumulative displacement:

$$
D(n, m, i)=\sum_{j=1}^{i} d(n, m, j)
$$

Before motion compensation, tissue residuals are removed from the contrast data by subtracting the background image obtained with a moving average filter of length $G_{b c k}$. The motion compensation is performed by shifting and spline interpolating the $i^{\text {th }}$ frame $\mathbf{I}_{c}(o, p, i)$ by $D(n, m, i)$ resulting in an image denoted $\mathbf{I}_{m c}(O, P, i)$. The SNR of the MBs is increased by attenuating high frequency noise using a low pass FIR filter of length $G_{l p}$ and a normalized cutoff frequency of $w_{c}$ providing the final images $\mathbf{I}_{p}(O, P, i)$ used for MB tracking.

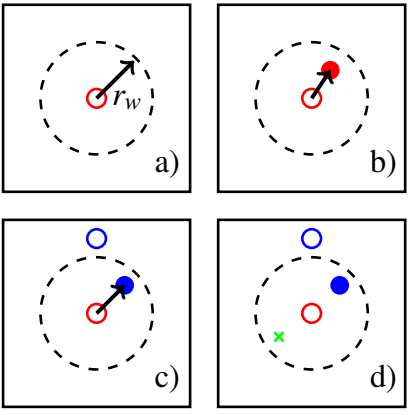

Fig. 2. Illustration of four scenarios when tracking a MB. An empty circle represent a MB in frame $i-1$ whereas a filled circle a bubble in frame $i$. A color relates to a specific bubble. The cross represent noise, which is mistakenly interpreted as being a $\mathrm{MB}$.

\section{Microbubble Tracking}

Individual MBs can be tracked through time as they move inside the blood system. This has been achieved with various MB tracking algorithms [1], [2], [8], [9]. An individual MB appears in the image as the PSF determined by the imaging system. The method uses the intensity peaks of the PSF to located MBs in each frame. Regions with intensities above a threshold of $I_{t h}$ are considered a MB. The center position is estimated by calculating the centroid of the PSF full-width-athalf-maximum (FWHM).

Reconnecting MBs in subsequent frames is performed using a search area at the old MB positions. The radius in the search area $\left(r_{w}\right)$ is based on the maximum expected velocity of the blood flow. A connection is made if a single MB is inside the area. Fig. 2 illustrates four possible situations, when trying to reconnect a bubble. An empty marker represent a MB in frame $i$, and a filled marker a bubble in frame $i+1$. Different colors relates to specific bubbles. A cross represent noise, which is interpreted as being a MB. The different situations from Fig. 2 are: a) old MB disappear and no connection is made. b) old MB reappear and correct connection made. c) old MB disappear and a new $\mathrm{MB}$ appears resulting in a wrong connection. d) Several MBs (false or valid) appear and no connection is made. The MBs with highest intensity is connected first, continuing until no more bubbles are left. The remaining unconnected MBs are saved to be tracked in the following frame.

Several instances can result in wrong connections. An indication of a valid MB is how consistent it is tracked. MBs tracked for less than a threshold of $t_{\min }$ are removed.

\section{METHODS}

\section{A. Equipment}

A modified ${ }^{1}$ BK3000 scanner from BK Ultrasound was used with a X18L5s Hockey stick transducer. Data were acquired with two different ultrasound sequences at different time instances during the experiment. A contrast sequence using an amplitude modulation techniques to excite the microbubbles in an nonlinear regime (which attenuate the tissue

\footnotetext{
${ }^{1}$ The scanner was modified to allow streaming of beamformed envelope detected data to a hard drive.
} 
signal [10], [11]) and a standard B-mode sequence used to display the tissue. The frame rate in both sequences was 50 $\mathrm{Hz}$. The axial and lateral resolution obtained with the B-mode sequence was: $\Delta l_{a x, b}=25.5 \mu \mathrm{m}, \Delta l_{l a t, b}=51 \mu \mathrm{m}$ and with the contrast sequence: $\Delta l_{a x, c}=43 \mu \mathrm{m}, \Delta l_{l a t, c}=197 \mu \mathrm{m}$.

Sonovue from $\mathrm{Bracco}^{2}$ was used as contrast agent, diluted with saline to get the desired concentration of MBs. A concentration of $1 / 10$ compared to standard applications for humans was used performing the experiment.

Table I provide a list of parameters used for motion compensation and tracking of MBs.

Table I

TABLE WITH PARAMETERS USED FOR MOTION COMPENSATION AND TRACKING OF MBS.

\begin{tabular}{cc}
\hline Parameter & Value \\
\hline Motion & Compensation \\
\hline$K$ & $100(4.3 \mathrm{~mm})$ \\
$G_{b c k}$ & 100 \\
$G_{l p}$ & 50 \\
$w_{c}$ & 0.3 \\
\hline
\end{tabular}

\begin{tabular}{|c|c|}
\hline Parameter & Value \\
\hline \multicolumn{2}{|c|}{ Tracking Algorithm } \\
\hline$r_{w}$ & $300 \mu \mathrm{m}$ \\
\hline$I_{t h}$ & $\max \left[\mathbf{I}_{M B}\right]-2 d B^{3}$ \\
\hline \multicolumn{2}{|c|}{ Resolution after Interpolation } \\
\hline$\Delta l_{a x, i n t}$ & $10 \mu \mathrm{m}$ \\
\hline$\Delta l_{\text {lat,int }}$ & $10 \mu \mathrm{m}$ \\
\hline
\end{tabular}

\section{B. In Vivo Experiment}

A Sprage-Dawley rat was used for the in vivo experiment The rat was anesthetized using isoflurane and the surrounding organs around the kidney was displaced to make space for the transducer. Injection of MBs into the kidney was prepared by inserting a cannula into the renal artery. The transducer was placed with contact to the exposed kidney and adjusted using a Power Doppler sequence as shown in Fig. 3.

During the experiment, the rat was connected to a mechanical ventilator with a fixed respiration cycle of 72 per minute. The MB dilution were infused directly into the renal artery while images were acquired using the contrast enhancing sequence. Immediately afterwards, the B-mode sequence was used to acquire images of the tissue movement. In this experiment, the movement was isolated purely to respiration (cycle period fixed mechanically) and pulse (controlled by anatomy).

\section{RESULTS}

At Fig. 3, a B-mode and a Doppler image of the kidney is presented. The red square indicates the area used to calculating the axial motion $D(n, m, i)$ shown in Fig. 5 and the tracking example shown in Fig. 6. Fig. 5 shows the axial displacement with and without contribution from the pulse, which is removed with a low pass filter. The plots are obtained by averaging 50 respiration cycles resulting in a standard deviation of $\sigma_{m c}=1.7$ $\mu \mathrm{m}$ after low pass filtering.

\footnotetext{
${ }^{2} \mathrm{http}: / /$ www.braccoimaging.com

${ }^{3} \mathbf{I}_{M B}$ is the intensity average of the microbubbles during the experiment.
}
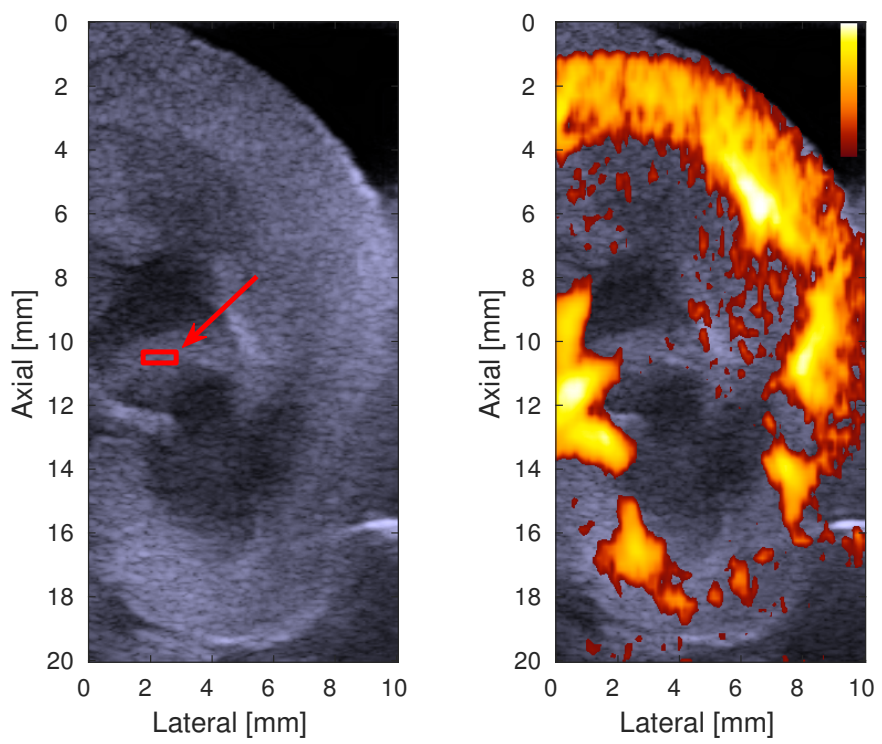

Fig. 3. [Left] - B-mode image showing the kidney. The red square indicates the area used for motion- and MB tracking shown in Fig. 6. [Right] - Power Doppler image of the kidney, where colored areas indicates blood flow.
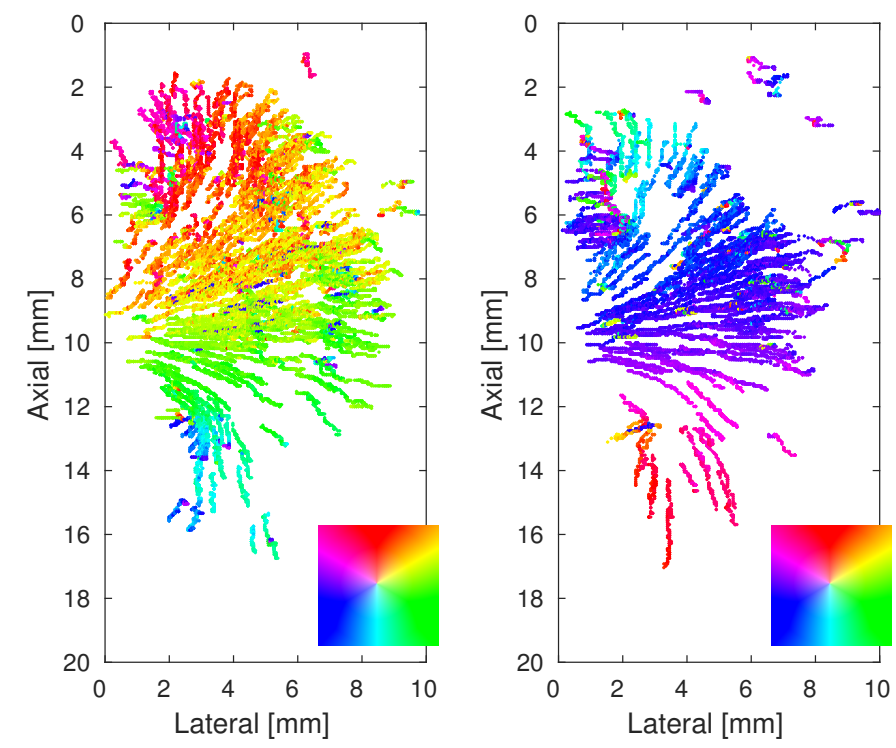

Fig. 4. [Left] - MB tracking image showing MBs flowing away from the kidney entrance set to be located at a depth of around $10 \mathrm{~mm}$. [Right] - Shows MBs flowing towards the entrance of the kidney. The color represent the direction given by the color-wheel.
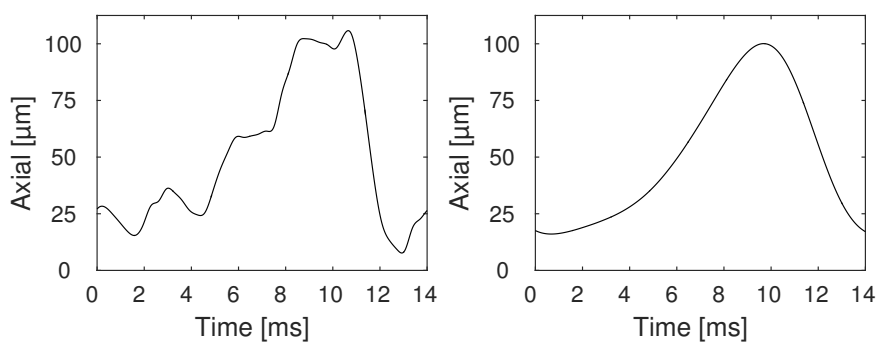

Fig. 5. [Left] - Axial displacement from the red square in Fig. 3, obtained by averaging 50 respiration cycles. [Right] - Axial displacement without contribution from pulse. 

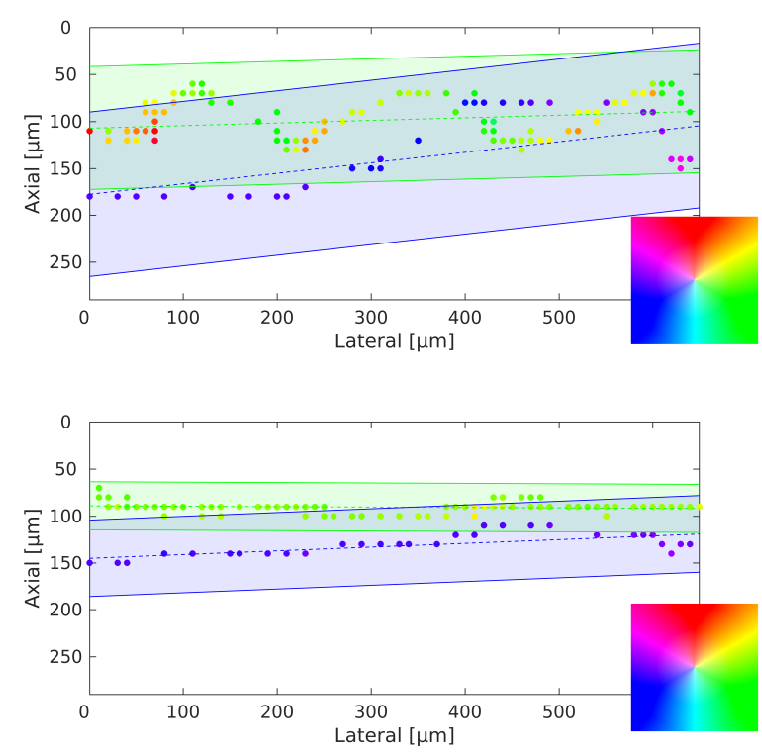

Fig. 6. [Top] - Shows two MBs tracked without motion compensation in the area shown in Fig. 3. [Bottom] - Result of tracking with motion compensation applied in the axial direction. Width of colored area correspond to $\pm 3 \sigma$.

Fig. 6 shows an example of two MBs tracked in the area highlighted in Fig. 3. The bottom figure illustrate the difference when compensating for the motion related to respiration (right plot in Fig. 5). A marker indicates the position of a located MB, and the color indicates its direction given by the color-wheel. By assuming the vessels to be straight within the small area, the standard deviation has been calculated. Before compensation (top figure): $\sigma_{n m c, g}=22 \mu \mathrm{m}$ for the green and $\sigma_{n m c, b}=34$ $\mu \mathrm{m}$ for the blue MB. After compensation (bottom figure): $\sigma_{m c, g}=8 \mu \mathrm{m}$ and $\sigma_{m c, g}=13.5 \mu \mathrm{m}$. The width of the colored areas correspond to $\pm 3 \sigma$.

Fig. 4 shows two MB tracking images of the entire kidney. The left and right image shows MBs flowing away and towards the entrance of the kidney set to be located at a depth of around $10 \mathrm{~mm}$. The images are created from data acquired over a period of $10 \mathrm{~min}$. After discarding MBs tracked for less than $2 \mathrm{~s}$, the total number of individual MBs are 432 and 237 for the left and right image respectively. In total, it resulted in 72.000 and $35.000 \mathrm{MB}$ localizations, each plotted in the figure with a color related to the direction.

\section{DISCUSSION}

The results presented provide a proof of concept of making in vivo super resolution images with non-fixated tissue. The axial motion related to the respiration could be estimated with a standard deviation of $\sigma_{m c}=1.7 \mu \mathrm{m}$, and performing compensation clearly yields a more realistic bubble motion. Relaxing the strict requirements of fixated tissue increases the feasibility of the technique significantly in a clinical setup.

This study is based on an initial in vivo experiment, and several improvements can be made to improve the results. The tracking algorithm is heavily dependent on a strong response from the MBs. A low SNR was obtained with the contrast sequence and future refinement is required. The low SNR gave rise to many false $\mathrm{MB}$ interpretations due to noise, which disturbed the tracking of valid MBs.

The B-mode and contrast data were not acquired simultaneous during the experiment. Better compensation can be obtained with direct frame to frame compensation.

When tracking MBs, it is important that they are spatially separated. Spatial close MBs will have overlapping PSFs. This significantly effect the appearance of the MBs and makes it hard to distinguish between them. One way to accommodate this separation is by infusing only a sparse dilution of MBs.

When imaging with a one-dimensional transducer, the MBs risk moving out of the imaging plane. Out of plane MBs can occur either from vessels not contained inside the imaging plane or due to tissue movement perpendicular to the plane. Thus, further studies should ideally be performed in 3-D.

\section{CONCLUSION}

This paper demonstrated super resolution imaging in a high density vascular structure using microbubbles in non-fixated tissue. It has been shown that the motion due to respiration from a rat kidney can be found with a high precision around $\sigma_{m c}=1.7 \mu \mathrm{m}$. Motion tracking has been used to compensate and align the vessels in contrast data containing only the responses from the microbubbles. Improvements were clearly shown by comparing tracking with and without compensation. Ultimately, this shows that the strict restriction of completely fixated tissue can be relaxed, when making super resolution imaging with microbubbles.

\section{REFERENCES}

[1] C. Errico, J. Pierre, S. Pezet, Y. Desailly, Z. Lenkei, O. Couture, and M. Tanter, "Ultrafast ultrasound localization microscopy for deep superresolution vascular imaging," Nature, vol. 527, pp. 499-502, November 2015.

[2] K. Christensen-Jeffries, R. J. Browning, M. Tang, C. Dunsby, and R. J. Eckersley, "In vivo acoustic super-resolution and super-resolved velocity mapping using microbubbles," IEEE Trans. Med. Imag., vol. 34, no. 2, pp. 433-440, February 2015.

[3] I. Akiyama, N. Nakajima, and S. Yuta, "Movement analysis using B-mode images," in Acoust. Imaging, vol. 17, 1988, pp. 499-505.

[4] J. S. Jeong, J. S. Hwang, M. H. Bae, and T. K. Song, "Effects and limitations of motion compensation in synthetic aperture techniques," in Proc. IEEE Ultrason. Symp., vol. 2, 2000, pp. 1759-1762.

[5] K. L. Gammelmark and J. A. Jensen, "Duplex synthetic aperture imaging with tissue motion compensation," in Proc. IEEE Ultrason. Symp., 2003, pp. 1569-1573.

[6] B. Y. S. Yiu, I. K. H. Tsang, and A. C. H. Yu, "A modified synthetic aperture imaging approach with axial motion compensation," in Proc. IEEE Ultrason. Symp., 2008, pp. 1254-1257.

[7] K. L. Gammelmark and J. A. Jensen, "2-D tissue motion compensation of synthetic transmit aperture images," IEEE Trans. Ultrason., Ferroelec., Freq. Contr., pp. 594-610, April 2014.

[8] W. G. Wilkening, J. Lazenby, and H. Ermert, "A method for detecting echoes from microbubble contrast agents based on time-variance," Proc. IEEE Ultrason. Symp., vol. 2, pp. 1823-1826, 1998.

[9] D. Ackermann and G. Schmitz, "Detection and tracking of multiple microbubbles in ultrasound B-mode images," IEEE Trans. Ultra., vol. 63, no. 1, pp. 72-82, January 2016.

[10] P. J. Phillips, "Contrast pulse sequences (CPS): imaging nonlinear microbubbles," Proc. IEEE Ultrason. Symp., pp. 1739-1745, 2001.

[11] R. J. Eckersley, C. T. Chin, and P. N. Burns, "Optimising phase and amplitude modulation schemes for imaging microbubble contrast agents at low acoustic power," Ultrasound Med. Biol., vol. 31, no. 2, pp. 213 219, 2005. 